\title{
Tratamiento endoscópico de la litiasis pieloureteral mediante láser Holmium-YAG. Dos años de experiencia
}

\author{
J.L. Palmero Martí, A. Budía Alba, J.L. Pontones Moreno, E. Broseta Rico, F. Boronat Tormo, \\ J.F. Jiménez Cruz
}

Servicio de Urología. Unidad de Litotricia y Endourología. Hospital Universitario La Fe. Valencia.

Actas Urol Esp 2005; 29 (6): 587-592

\section{RESUMEN}

TRATAMIENTO ENDOSCÓPICO DE LA LITIASIS PIELOURETERAL MEDIANTE LASER HOLMIUM-YAG. DOS AÑOS DE EXPERIENCIA

Objetivo: Demostrar la eficacia y seguridad del láser Holmium-YAG en el tratamiento de litiasis pieloureterales, de acuerdo con la experiencia acumulada en nuestro centro desde la introducción de esta fuente de energía.

Material y métodos: Entre Enero del 2002 y Febrero del 2004 realizamos 198 ureterorrenoscopias utilizando un láser Holmium para el tratamiento de litiasis localizadas en el tracto urinario superior. En todos los casos el diagnóstico se realizó mediante un estudio radiológico previo. Evaluamos la eficacia del procedimiento mediante una radiografía simple a las 3-4semanas de la intervención, considerándolo eficaz si los restos litiásicos eran menores de $2 \mathrm{~mm}$ o inexistentes. Analizamos la localización y las características de las litiasis, sus aspectos técnicos, resultados y complicaciones de nuestra serie.

Resultados: La localización más frecuente de las litiasis fue el uréter pélvico (59\%), siendo similar el número de casos tratados tanto en uréter iliaco como lumbar (16\%). Un 70\% de las litiasis tenían un diámetro mayor entre 0,5 y $1,5 \mathrm{~cm}$, y el 15\% eran mayores de 1,5 cm. En el 61,8\% de los casos, la litotricia se realizó con raquianestesia, siendo la media de pulsos utilizados para la litofragmentación en la serie de 2532 (intervalo confianza 95\%) utilizando una potencia de trabajo de $1 \mathrm{~J}$ en todos los casos. La eficacia global del procedimiento en función del tamaño y localización de las litiasis fue del 95,5\%. La tasa de complicaciones fue escasa $(2,19 \%)$.

Conclusiones: La litofragmentación con láser Holmium-YAG es un procedimiento eficaz y seguro ya que, en nuestra experiencia, consigue una eficacia del $95,5 \%$ en el tratamiento de litiasis pielo-ureterales con escasas complicaciones.

Palabras clave: Litiasis, Litofragmentación, Láser Holmium-YAG.

\section{ABSTRACT \\ ENDOSCOPIC TREATMENT OF PIELO-URETHERAL LITHIASIS WITH HOLMIUM-YAG LASER. TWO YEARS OF EXPERIENCE}

Objective: To show the efficiency and safety of Holmium laser in the treatment of pyeloureteral lithiasis, based on our own experience since the introduction of this source of energy in our department.

Material and methods: From January 2002 to February 2004, we have carried out 198 ureterorenoscopies using Holmium laser to treat lithiasis located in the upper urinary tract. In all cases, a previous radiological study demonstrated the stone. Follow-up, where the efficacy of the treatment was assessed, was done with a scout $\mathrm{x}-$ ray after 3-4 weeks. We evaluated the localization and features of the lithiasis, technical aspects, results and complications of our series.

Results: The most common localization of the lithiasis was the pelvic ureter (59\%), with the number of treated cases in both the iliac and lumbar ureter being similar (16\%). $70 \%$ of the lithiasis had a size between 0.5 and $1.5 \mathrm{~cm}$, and $15 \%$ showed a diameter bigger than $1.5 \mathrm{~cm}$ In $61.8 \%$ of cases, laser lithotripsy was carried out under spinal anaesthesia, being the average of the pulses used 2532 (confidence interval 95\%), using a working power of $1 \mathrm{~J}$ in all cases. The overall efficacy of the procedure in terms of size and localization was 95.5\%. The complication rate was scarce $(2.19 \%)$.

Conclusions: Holmium laser lithotripsy is a very effective and safe procedure since in our experience it is 95.5\% efficient for pyeloureteral lithiasis treatment and has a low rate of complications.

Keywords: Lithiasis. Lithofragmentation. Holmium laser. 
$\mathrm{E}$ abordaje terapéutico de las litiasis pieloureterales comprende un amplio abanico de posibilidades, que van desde la clásica litotricia con ondas de choque a otras técnicas como la nefrolitotomía percutánea y las técnica endourológicas con ureterorrenoscopia, que emplean fuentes de energía diversa para la fragmentación del cálculo.

La experiencia cada vez mayor en el uso del ureteroscopio y el calibre progresivamente menor de estos, junto con la aparición de nuevas fuentes de energía, convierte la litofragmentación endoscópica de las litiasis pieloureterales en un procedimiento eficaz, seguro y de primera línea en el tratamiento de esta patología ${ }^{1,2}$. Todo lo expuesto, unido al creciente desarrollo de la laparoscopia en urología, evidencia que la cirugía abierta de la litiasis cuente con indicaciones muy limitadas en el momento actual. Sin embargo, la laparoscopia no es todavía una técnica de primera línea en esta patología, aunque sí puede sustituir a la cirugía abierta cuando fracasan el resto de opciones mencionadas con anterioridad ${ }^{3,4}$.

La utilización del láser Holmium-YAG comienza a principios de la década de los noventa ${ }^{5}$ demostrando su eficacia en litofragmentación en un estudio experimental realizado por Sayer et al en $1993^{6}$. A lo largo de esta década, también se ha demostrado su utilidad en el tratamiento de diversas patologías urológicas merced a una serie de características propias. Por un lado, la absorción energética por el agua en superficie al contacto con la fuente de energía, produce una fotovaporización microscópica ${ }^{7-9}$, permitiendo la fragmentación de las litiasis desde la superficie hacia el núcleo, siendo útil en litiasis de cualquier composición ${ }^{8,10,11}$. De otra parte, su capacidad de utilización como energía que permite realizar corte y coagulación, lo convierten en una fuente versátil útil en el tratamiento de otros procesos como por ejemplo tumores del tracto urinario superior, estenosis de la vía urinaria, etc... (endopielotomía endoscópica en el síndrome de la unión pieloureteral) ${ }^{11}$.

Dadas estas características, la utilización del láser Holmium-YAG para el tratamiento de litiasis pieloureterales ha demostrado ser un procedimiento eficaz y seguro en las distintas localizaciones del cálculo ${ }^{10,12}$.
En este trabajo analizamos la eficacia del láser Holmium-YAG en el tratamiento de litiasis pieloureterales tras dos años de experiencia en nuestro centro.

\section{MATERIAL Y MÉTODOS}

Hemos realizado una revisión prospectiva, que incluye 198 procedimientos endoscópicos en los que se empleó como fuente de energía el láser Holmium-YAG, durante el periodo comprendido entre Enero del 2002 y Enero del 2004, con el objetivo de evaluar la eficacia de esta fuente de energía en el tratamiento de litiasis del tracto urinario superior.

Las indicaciones para la litofragmentación con Holmium-YAG fueron: en el caso del varón, cualquier localización de la litiasis tras fracasar la litofragmentación con tres sesiones de ondas de choque. En el caso de la mujer, las litiasis localizadas en los segmentos pélvico o iliaco del uréter fueron consideradas como indicaciones primarias para el procedimiento, mientras que en el caso del uréter lumbar, se tomaron las mismas consideraciones que en el caso del varón.

La fuente de energía que utilizamos en la litofragmentación fue un láser de cuerpo sólido tipo Holmium-YAG (Dornier-Medilas ${ }^{\circledR}$ ), que como principales características técnicas emplea longitudes de onda y frecuencias de trabajo variables; entre 2080 y $2110 \mathrm{~nm}$ y $5-30 \mathrm{~Hz}$ respectivamente. Además este tipo de fuente emite energía en forma de pulsos de duración fija $(250 \mu \mathrm{s})$ y con intensidad o potencia de trabajo regulable, pudiendo emplear intensidades entre 200 y 1800 $\mathrm{mJ}$, lo cual permite su empleo en el tratamiento de otras patologías.

Para la transmisión de la energía utilizamos fibras de cuarzo flexibles (Dornier®) de diámetro variable entre 350 y $550 \mu \mathrm{m}$, empleando en todos los casos un ureterorrenoscopio semirrígido telescopado (Stortz ${ }^{\circledR}$ ), con un calibre de 7,2 F en su extremo distal, trabajando con irrigación continua de suero fisiológico que es infundido con bomba para el preciso control de la presión/flujo a lo largo de todo el procedimiento.

Todos los procedimientos se realizaron bajo anestesia, siendo la raquianestesia la técnica más empleada (62\%), realizando sedación en un $21 \%$ de los casos y en el resto (17\%) se procedió 
a una anestesia general. La profilaxis antibiótica utilizada en la mayoría de los casos consistió en dos dosis de $100 \mathrm{mg}$ de tobramicina intravenosa una hora antes del procedimiento y a las ocho horas de la manipulación, excepto en los casos de contraindicación o alergia medicamentosa en los que se sustituyó por una cefalosporina de tercera generación siguiendo la misma pauta.

Evaluamos la eficacia del procedimiento, mediante una radiografía simple que se realizó a las tres semanas del procedimiento. Se consideraron eficaces aquellos casos en los que en la radiografía de control no existían restos litiásicos o bien éstos eran de tamaño inferior a $2 \mathrm{~mm}$ y por tanto presumiblemente expulsables.

Analizamos en la serie: localización de las litiasis, indicación, número de pulsos en función del tamaño y necesidad de doble J. Evaluamos la eficacia de la URS y litofragmentación con láser Holmium-YAG en función de los criterios anteriores. La valoración estadística de estos se realizó mediante el test estadístico Chi cuadrado.

\section{RESULTADOS}

La localización más frecuente de las litiasis tratadas con láser Holmium-YAG fue el uréter pélvico (59\% de los casos), siendo idéntica la frecuencia en las localizaciones iliaca y lumbar (16\%). En el resto de los casos, la localización fue piélica (2\%) y en un $7 \%$ de casos de la serie se trataron "calles litiásicas" derivadas de litotricias extracorpóreas previas con este procedimiento de litofragmentación.

En el 61,3\% de los casos, la indicación de litofragmentación Holmium-YAG fue primaria, mientras que, en el resto se realizó tras fracaso del tratamiento con ondas de choque $(33,7 \%)$ o tras fracaso en un intento de fragmentación previo con este mismo procedimiento (5\%).

En todos los casos, la potencia de trabajo que empleamos para la litofragmentación fue de $1 \mathrm{~J}$, mientras que, la frecuencia de pulsos de energía fue variable entre 5 y $10 \mathrm{~Hz}$. La media de pulsos empleados en los 198 casos fue de 2.532 (intervalo de confianza del 95\%), siendo la relación número de pulsos-tamaño de la litiasis estadísticamente significativa al realizar un análisis con Chi cuadrado $(p<0,05)$. Las litiasis se han clasificado en cuatro grupos en función de su diámetro longitudinal en centímetros $(<0,5,0,5-1,1-1,5$, $>1,5$ ). Mostramos los resultados obtenidos en cuanto al número de pulsos necesarios para la fragmentación de la litiasis y el tamaño de las mismas (Tabla 1).

\section{Tabla 1}

Relación no pulsos / tamaño litiasis

\begin{tabular}{ccccc}
\hline $\begin{array}{c}\text { № pulsos/ tamaño }(\mathrm{cm}) \\
\%\end{array}$ & $<0,5$ & $0,5-1$ & $1-1,5$ & $>1,5$ \\
\hline$<500$ & 25 & 14,1 & - & - \\
$500-2500$ & 66,7 & 63,4 & 34 & 25,7 \\
$>2500$ & 8,3 & 22,5 & 66 & 74,3 \\
\hline
\end{tabular}

Chi cuadrado: 51,21 p 0,024

El $61,6 \%$ de los pacientes sometidos a este procedimiento requirieron de catéter doble $\mathrm{J}$ tras el tratamiento, siendo el tiempo medio hasta la retirada del mismo de 23,58 días. Las indicaciones de colocación del doble $J$ tras la litofragmentación fueron impactación de la litiasis, edema de la pared ureteral o presencia de fragmentos litiásicos no expulsables al terminar el procedimiento.

Evaluamos la eficacia del procedimiento, en función de la localización de las litiasis y el tamaño de las mismas según los hallazgos en el control radiológico realizado a las tres semanas del tratamiento. La eficacia del procedimiento en función de la localización osciló entre el 90,7\%, para las litiasis de uréter lumbar, y el 100\% para las de uréter pélvico y piélicas, siendo los resultados estadísticamente significativos (Chi cuadrado: $33,13, \mathrm{p}<0,05)$. Hay que señalar que la alta eficacia en las litiasis piélicas se debe a que fueron casos seleccionados con litiasis de pequeño tamaño (menores de un centímetro) y fácilmente accesibles, representando estos casos tan solo el $2 \%$ del total ( 4 casos). De otra parte, en el caso de litiasis de alta complejidad o "calles litiásicas", que representan un $7 \%$ de la serie (14 enfermos), la eficacia fue del $73,7 \%$. Por tanto, la eficacia global del procedimiento atendiendo a los resultados según localizaciones fue del 95,5\%. Presentamos una tabla con los resultados de eficacia según localización (Tabla 2).

De la misma manera, al evaluar la eficacia en función del tamaño de la litiasis obtuvimos resultados que oscilan entre $97,8 \%$ para litiasis de menos de $0,5 \mathrm{~cm}$ al $92,3 \%$ para las mayores de 
Tabla 2

Eficacia según localización de la litiasis

\begin{tabular}{lccccc}
\hline $\begin{array}{l}\text { Localización/ } \\
\text { Restos RX. }\end{array}$ & $\begin{array}{c}\text { Pélvico } \\
(\%)\end{array}$ & $\begin{array}{c}\text { Iliaco } \\
(\%)\end{array}$ & $\begin{array}{c}\text { Lumbar } \\
(\%)\end{array}$ & $\begin{array}{c}\text { Piélica } \\
(\%)\end{array}$ & $\begin{array}{c}\text { Calle } \\
\text { Litiasica } \\
(\%)\end{array}$ \\
\hline RX Negativa & $\mathbf{6 6 , 7}$ & $\mathbf{6 6}$ & $\mathbf{6 0 , 5}$ & $\mathbf{6 6 , 6}$ & $\mathbf{3 1 , 6}$ \\
$<2 \mathrm{~mm}$ & $\mathbf{3 3 , 3}$ & $\mathbf{3 2}$ & $\mathbf{3 0 , 2}$ & $\mathbf{3 3 , 4}$ & $\mathbf{4 2 , 1}$ \\
$>2 \mathrm{~mm}$ & - & 2 & 9,3 & - & 26,3 \\
Eficacia & $\mathbf{1 0 0}$ & $\mathbf{9 8}$ & $\mathbf{9 0 , 7}$ & $\mathbf{1 0 0}$ & $\mathbf{7 3 , 7}$ \\
\hline
\end{tabular}

Chi cuadrado: 33,13 p 0,005

$1,5 \mathrm{~cm}$ siendo de nuevo los resultados estadísticamente significativos (Chi cuadrado: 31,22, $\mathrm{p}<0,05)$, con una eficacia global del procedimiento según los resultados en función del tamaño de la litiasis del 95,17\%. Recogemos los resultados de la eficacia en función del tamaño de la litiasis en una tabla (Tabla 3).

\section{Tabla 3}

Eficacia según tamaño de las litiasis

\begin{tabular}{lcccc}
\hline $\begin{array}{l}\text { Tamaño(cm)/ } \\
\text { Restos RX. }\end{array}$ & $\begin{array}{c}<0,5 \\
(\%)\end{array}$ & $\begin{array}{c}0,5-1 \\
(\%)\end{array}$ & $\begin{array}{c}1-1,5 \\
(\%)\end{array}$ & $\begin{array}{c}>1,5 \\
(\%)\end{array}$ \\
\hline RX Negativa & $\mathbf{8 1 , 5}$ & $\mathbf{7 0 , 4}$ & $\mathbf{5 1 , 4}$ & $\mathbf{3 5 , 9}$ \\
$<2 \mathrm{~mm}$ & $\mathbf{1 6 , 3}$ & $\mathbf{2 7}$ & $\mathbf{4 2 , 9}$ & $\mathbf{5 6 , 4}$ \\
$>2 \mathrm{~mm}$ & 2,2 & 2,6 & 5,7 & 7,7 \\
Eficacia & $\mathbf{9 7 , 8}$ & $\mathbf{9 7 , 4}$ & $\mathbf{9 4 , 3}$ & $\mathbf{9 2 , 3}$ \\
\hline
\end{tabular}

Chi cuadrado: 31,22 p 0,005

Las complicaciones que hemos apreciado han sido escasas, representando el 2,19\% de los casos tratados con este procedimiento. Cabe reseñar, tres casos en los que se produjeron laceraciones ureterales importantes por la dificultad del procedimiento, que se resolvieron con un mayor tiempo de permanencia del catéter doble $J$, una rotura y migración ascendente de una guía hidrófila que pudo extraerse en el mismo acto endoscópico y una bacteriemia que generó un cuadro de shock séptico, precisando el paciente ingreso en la Unidad de Cuidados Intensivos.

\section{DISCUSIÓN}

El tratamiento de la litiasis constituye una de las parcelas de la Urología que se encuentra en constante evolución merced al desarrollo tecnológico que permite abordajes cada vez menos cruentos con óptimos resultados. Desde el desarrollo en la década de los ochenta de la litotricia con ondas de choque, que cambió el clásico abordaje quirúrgico de la litiasis ${ }^{10}$, los procedimientos endourológicos para el tratamiento de la litiasis, han ido ganando terreno merced a las mejoras técnicas del aparataje endoscópico ${ }^{13}$ y el desarrollo de fuentes de energía diversas para litofragmentación, unido a la mayor experiencia en el manejo del ureteroscopio en algunos centros.

El Holmium-YAG fue utilizado con buenos resultados en Oftalmología y Otorrinolaringología antes de su utilización en Urología en 1993 por Webb et $\mathrm{al}^{14}$. Esta herramienta es un dispositivo fotolumínico de cuerpo sólido que emite pulsos energéticos con una longitud de onda de 2.100 $\mathrm{nm}$, próxima al espectro de la luz infrarroja lo cual la hace imperceptible para el ojo huma$\mathrm{no}^{8,10}$. Por otro lado tiene una gran versatilidad, ya que permite trabajar con intensidades y frecuencias variables $(0,2-2,8 \mathrm{~J}, 5-30 \mathrm{~Hz})$, lo cual permite su empleo para el tratamiento de patología urológicas diversas, ya que, es posible su utilización como dispositivo de corte y fotocoagulación $^{8}$. De otra parte el láser Holmium-YAG permite emplear fibras de transmisión lumínica de diámetros variables, entre 200 y $1000 \mu \mathrm{m}^{8,15}$. El empleo de fibras de reducido calibre (200-365 $\mu \mathrm{m})$ facilita el acceso a cualquier localización de la vía urinaria, ya que, permite su uso tanto con ureteroscopios rígidos como flexibles ${ }^{1}$. En nuestro centro, utilizamos habitualmente para este procedimiento fibras de cuarzo de calibres 365$550 \mu \mathrm{m}$, ya que utilizamos habitualmente un ureteroscopio semirrígido, reservando el flexible para casos de extrema dificultad por angulaciones ureterales extremas, bucles de uréter lumbar o para el acceso a las cavidades renales.

A esta longitud de onda, la energía pulsátil es absorbida por el agua en la superficie de contacto de la fibra lumínica. Debido a esto, se ha demostrado en estudios de eficacia "in vitro", que el láser Holmium-YAG tiene una capacidad de corte y coagulación "superior a la de otras energías lumínicas ${ }^{16}$. En el caso de las litiasis, la absorción en superficie produce una fragmentación de la piedra desde la periferia hasta el núcleo, siendo útil en el tratamiento de todo tipo de litiasis independientemente de su composición ${ }^{7,9,10,15,17,18}$. 
Otra de las características del Holmium-YAG a señalar es que la retropulsión de los fragmentos litiásicos es inferior a la de otros láseres, además los fragmentos obtenidos son de menor tamaño y las fibras de transmisión que generalmente se utilizan son de menor calibre como hemos comentado. Por ello, las posibles complicaciones por la migración de los fragmentos se ven reducidas ${ }^{19}$.

De otra parte, la penetrabilidad tisular es menor a la de otros láseres, siendo ésta inferior a 0, 5 milímetros desde la superficie de aplicación, con lo que la probabilidad de perforaciones ureterales es menor ${ }^{8}$.

Por todo lo expuesto, el Holmium-YAG es una fuente energética segura, siendo el riesgo de complicaciones bajo, entre el 1-5\% de los casos según series ${ }^{1,10,17,20}$. El riesgo se reduce por las propias características físico-químicas de la fuente energética y la posibilidad de emplear endoscopios y fibras de conducción de calibres menores. Los resultados obtenidos en nuestra serie se ajustan a la literatura, ya que las complicaciones durante el procedimiento han sido escasas $(2,19 \%$ casos), destacando tan sólo tres casos con laceraciones o perforación ureteral $(1,51 \%)$, una rotura-migración de guía durante la ureterorrenoscopia que se solventó en el mismo acto y una bacteriemia tras la instrumentación que precisó de ingreso en la Unidad de Cuidados Intensivos.

Otros datos que avalan la seguridad en litofragmentación de esta fuente de energía son aportados por diversos autores que no desaconsejan su uso en el tratamiento de litiasis en circunstancias especiales, como en pacientes con diátesis hemorrágicas ${ }^{21}$, litiasis en la edad pediátrica o en el embarazo ${ }^{25}$, siempre y cuando se realice en centros con la suficiente experiencia en procedimientos endourológicos. En nuestro centro contamos con alguna experiencia en el tratamiento endoscópico de litiasis ureterales en niños, sin embargo, nuestra casuística es pequeña pero si podemos afirmar que la seguridad y la eficacia del Holmium-YAG en niños es similar al adulto.

En cuanto a la eficacia global del láser Holmium-YAG en litofragmen-

\section{Tabla 4}

tación, la mayoría de autores la sitúan por encima del 93\%, obteniéndose los mejores resultados en el tratamiento de litiasis ureterales bajas o de uréter pélvico-iliaco ${ }^{7,10,11,15,17,24}$. En este sentido, Lam et al. ${ }^{12}$ aconsejan la litofragmentación endoscópica, como primera opción terapéutica en litiasis de uréter proximal independientemente del tamaño. Este autor obtiene en su serie mejores resultados en cuanto a eficacia y seguridad, al comparar este procedimiento frente a las clásicas ondas de choque en las circunstancias descritas anteriormente. Los resultados obtenidos en nuestra serie, se ajustan a los que aparecen en la literatura, con una tasa de eficacia global del procedimiento de 95,5\%, según localización y tamaño, siendo incluso mejores en las localizaciones anteriormente descritas como se puede observar en las Tablas 2 y 3.

Presentamos un resumen de los resultados obtenidos en litofragmentación con láser Holmium-YAG en algunas de las series analizadas en esta revisión según la localización de la litiasis (Tabla 4).

$\mathrm{Al}$ analizar los resultados constatamos que, en el $62 \%$ de nuestros pacientes se ha considerado necesario la colocación de un catéter doble $J$ tras el procedimiento. En nuestra experiencia, lo empleamos en aquellos casos en los que se evidencie incrustación significativa de la litiasis o edema llamativo asociado, así como si tras el procedimiento persisten fragmentos presumiblemente no expulsables ( $>2$ milímetros), con el fin de evitar posibles complicaciones. En este sentido queremos reseñar que nuestra tendencia en el tiempo se ha ido modificando. Al inicio de utilizar esta técnica ureterorrenoscopia y catéter doble $\mathrm{J}$ eran nuestra norma para evitar complicaciones, pero la experiencia acumulada en más de cinco mil procedimientos nos ha hecho cada vez más

Eficacia en litofragmentación del Holmium-YAG

\begin{tabular}{lcccccc}
\hline Serie & $\mathbf{n}$ & $\begin{array}{c}\text { Renal } \\
\text { (\%) }\end{array}$ & $\begin{array}{c}\text { U. Iumbar } \\
\text { (\%) }\end{array}$ & $\begin{array}{c}\text { U iliaco } \\
\text { (\%) }\end{array}$ & $\begin{array}{c}\text { U pélvico } \\
\text { (\%) }\end{array}$ & $\begin{array}{c}\text { Global } \\
\text { (\%) }\end{array}$ \\
\hline Sofer & 598 & 84 & 97 & 100 & 98 & 97 \\
Devarajan & 300 & - & 89 & 96 & 97 & 93 \\
Grasso & 210 & 90 & 96 & 97 & 100 & 97 \\
Tawfiek & 155 & 87,3 & 96,5 & 100 & 100 & 95 \\
Shoroff & 100 & - & 81 & 99 & 98 & 93
\end{tabular}


estrictos en la utilización de estos catéteres, puesto que en muchos casos provocan mayores molestias que la propia expulsión de los fragmentos y requieren de una nueva endoscopia para su retirada.

\section{CONCLUSIONES}

La litofragmentación con láser Holmium-YAG es un procedimiento eficaz para el tratamiento de litiasis pieloureterales, independientemente de su composición. En nuestro centro hemos conseguido una eficacia global del procedimiento del $95,5 \%$, con escasas complicaciones $(2,19 \%)$, por lo que creemos que es éste un procedimiento eficaz y seguro. Por ello, consideramos que actualmente la litofragmentación endoscópica con láser Holmium-YAG constituye junto con a las clásicas ondas de choque, la primera línea de tratamiento en litiasis pieloureterales, considerándola en nuestra experiencia como de primera elección en localizaciones ureterales más distales y sexo femenino.

\section{REFERENCIAS}

1. Mugiya S, Ohhira T, Un-Ho T, Takayana T, Suzuki K, Fujita K. Endoscopic management of upper urinary tract disease using a $200 \mu \mathrm{m}$. Holmium laser fiber: Initial experience in Japan. Urology 1999;53(1):60-64.

2. Alapont JM, Broseta E, Pontones JL, Oliver F, Palmero JL, Jiménez Cruz JF. Complicaciones de la ureterorrenoscopia. Actas Urol Esp 2003;27(7):692-699.

3. Goel A, Hemal AK. Upper and mid-ureteric stones: a prospective unrandomized coparison of retroperitoneoscopic and open ureterolithotomy. BJU Int 2001;88(7):679-682.

4. Gaur DD, Trivedi S, Prabhudesai MR, Madhusudhana HR, Gopichand M. Laparoscopic ureterolithotomy : technical considerations and long-term follow-up. BJU Int 2002;89 (4):339-443.

5. Johnson DE, Cromeens DM, Price PR. Use of the Holmium: YAG laser in urology. Lasers Surg Med 1992;12:353-358.

6. Sayer J, Johnson DE, Price PR, Cromeens DM. Ureteral lithotripsy with Holmium-YAG laser. J Clin Laser Med Surg 1993;11:61-65.

7. Grasso M, Chalik Y. Principles and applications of laser lithotripsy: experience with the Holmim-YAG laser litothrite. J Clin Laser Med Surg 1998;16:3-7.

8. Baggley D, Erhard M. Use of the Holmium laser in the upper urinary tract. Techniques in Urology 1995;1:25-30.

9. Grasso M. Experience with the Holmium laser as an endoscopic lithotrite. Urology 1996;48(29):199-206.
10. Sofer M, Watterson JD, Wollin TA, Nott L, Razvdi H, Densdet JD. Holmium: YAG laser lithotripsy for upper urinary tract calculi in 598 patients. J Urol 2002;167:31-34.

11. Shroff S, Watson GM, Parikh A, Thomas R, Soonawalla PF, Pope A. The Holmium: YAG laser for ureteric stones. $\mathrm{Br} \mathrm{J}$ Urology 1998;78:836-839.

12. Lam JS, Greene TD, Gupta M. Treatment of proximal ureteral calculi: Holmium:YAG laser versus ureterolithotripsy versus extracorporeal shock wave litothripsy. J Urol 2002;167:1972-1975.

13. Grasso M, Bagley D. A 7,5/ 8,2 French actively deflectable, flexible ureteroscope: a new device for both diagnostic and therapeutic upper urinary tract endoscopy. Urology 1994; 43:435-439.

14. Webb DR, Kockelburgh R, Johnson WF. The vers-pulse Holmium surgical laser in clinical urology: a pilot study. Minin Invas Ther 1993;2:23-26.

15. Lembenger Devarajan R, Ashraf M, Beck RO, Lembenger RJ, Taylor MC. Holmium: YAG lasertripsy for ureteric calculi: an experience of 300 procedures. Br J Urol 1998;82: 342-347.

16. Wollin TA, Denstedt JD. The Holmium laser in Urology. J Clin Laser Med Surg 1998;16:13-19.

17. Leveille RJ, Lobik L. Intracorporeal lithotripsy: which modality is best? Curr Opin Urol 2003;13(3):249-253.

18. Shekhar C, Cornford PA, Powell CS. Ureteroscopic Holmium lasertripsy for ureteric stones. Scand J Urol Nephrol 1998;32:92-93.

19. Lee H, Ryan RT, Teichman JM, Kim J, Choi B, Arakeri NV, et al. Stone retropulsion during Holmium: YAG lithotripsy. J Urol 2003;169(3):881-885.

20. Santa-Cruz RW, Leveillee RJ, Krongrad A. Ex vivo comparison of four lithotripters commonly used in the ureter: what does it take to perforate? J Endourol 1998;12:417420 .

21. Watterson JD, Girvan AR, Cook AJ, Beiko DT, Nott L, Auge BK, et al. Safety and efficacy of Holmium: YAG laser lithotripsy in patients with bleeding diatheses. J Urol 2002;168 (2):442-445.

22. Wollin TA, Teichman JM, Rogenes VJ. Holmium: YAG lithotripsy in children. J Urol 1999;162:1717-1720.

23. Riddell JV, Denstedt JD. Management of urolithiasis during pregnancy. Contemp Urol 2002;1:12-16.

24. Tawfiek ER, Bagley DH. Management of upper urinary tract calculi with ureteroscopic techniques. Urology 1999; 53(1):25-30.

Dr. J.L. Palmero Martí

Servicio de Urología

Hospital Universitario La Fe

Avda. Campanar n 21

46009 Valencia

e- mail: joseluipalmer@hotmail.com

(Trabajo recibido el 3 febrero de 2005) 